\title{
THE PECULIAR BINARY SYSTEM HR 8891 (ET AND)
}

R. KUSCHNIG*†॰1, W. W. WEISS* ${ }^{* \circ 1}$, N. PISKOUNOV ${ }^{\dagger \circ 2}$, T. RYABCHIKOVA ${ }^{\text {to } 2}$, T.J. KREIDL ${ }^{* 3}$, and M. ALVAREZ ${ }^{* 4}$, S.G. BEDOLLA ${ }^{* 4}$, S.J. BUS ${ }^{* 3}$, Z. GUO ${ }^{* 5}$, J. HAO ${ }^{* 5}$, L. HUANG*5, F. KUPKA ${ }^{\dagger 1}$, D. Le CONTEL ${ }^{\circ 8}$, J.M. Le CONTEL ${ }^{\circ 8}$, D.J. OSIP ${ }^{* 3}$, K. PANOV ${ }^{* 6}$, N. POLOSUKCHINA*7, J.P. SAREYAN ${ }^{* 8}$, H. SCHNEIDER ${ }^{\circ 9}$, J.C. VALTIER ${ }^{\dagger 8}$, M. ZBORIL ${ }^{\circ 10}$, J. ŽIŽŇOVSKÝ ${ }^{* 10}$, J. ZVERKO*10

Contributions to photometry $\left({ }^{*}\right)$, spectroscopy $\left({ }^{\dagger}\right)$ and mapping $\left({ }^{\circ}\right)$

${ }^{1}$ Institute for Astronomy, Vienna, ${ }^{2}$ Astronomical Institute of the Russian Academy of Sciences, Moscow, ${ }^{3}$ Lowell Observatory, Flagstaff, ${ }^{4}$ Instituto de Astronomia - UNAM, Mexico ${ }^{5}$ Beijing Astronomical Observatory, ${ }^{6}$ Institute of Astronomy, Sofia,${ }^{7}$ Crimean Astrophysical Observatory, Ukraine, ${ }^{8}$ Observatoire de

la Côte d'Azur, Nice, ${ }^{9}$ Universitätssternwarte, Göttingen, ${ }^{10}$ Astronomical Institute of the Slovak Academy of Sciences, Tatranska Lomnica

\section{Introduction}

ET And is a binary system with a $\mathrm{B} 9 \mathrm{Si}$ star as the main component $\left(\mathrm{P}_{\text {orb }}=\right.$ $48.308^{\mathrm{d}}, \mathrm{e}=0.46$ ). Controversial claims in the literature concerning pulsation with periods ranging from few minutes to few hours and with variable amplitudes indicated a challenging target and motivated us to organize several photometric and spectroscopic observing campaigns. The problem with pulsation of ET And is that $T_{\text {eff }}$ and $\log g$ put this star in the cool domain of Slowly Pulsating B-type (SPB) stars, but the pulsation periods would be too short by a factor of about four, relatively to the shortest hitherto known periods for SPB stars.

\section{Rotation and pulsation}

Period and shape $\left(\mathrm{P}_{\text {rot }}=1.618^{\mathrm{d}}, \mathrm{a}_{\text {rot }}=23 \mathrm{mmag}(\mathrm{B})\right)$ of the rotation light curve did not change during the last three decades. Second, there are strong evidence that the short time scale variations attributed in the literature to ET And are due to pulsation of the main comparison star HD 219891 (HR 8870, A5 V). If this suspicion can be corroborted, a serious conflict between observation and current theory of stellar opacities will be eliminated and HD 219891 can be identified as a new $\delta$ Sct type variabel. Two pulsation periods $\left(\mathrm{P}_{1}=2.38^{\mathrm{h}}, \mathrm{a}_{1}=4.1 \mathrm{mmag}(\mathrm{B}), \mathrm{P}_{2}=3.55^{\mathrm{h}}, \mathrm{a}_{2}=1.8 \mathrm{mmag}(\mathrm{B})\right)$ are sufficient to reproduce the observed amplitude spectrum to a noise level of 0.3 mmag.

A full account on our photometry is being prepared for publication (Weiss et al. 1993, A\&A in prep.). 


\section{Atmospheric parameters and abundances}

The spectrum of ET And ranging from $4000 \AA$ to $7500 \AA$ was observed in 17 overlapping segments with the AURELIE Coudé spectrograph (resolution $=0.15 \AA$ ) of OHP. First, we tried to fit the observed Balmer line profiles ( $\mathrm{H} \alpha$ to $\mathrm{H} \delta$ ) with the help of Kurucz ATLAS 9 atmospheres and obtained an optimum fit for $T_{\text {eff }}=11500 \mathrm{~K}, \log \mathrm{g}=3.5$, and $\mathrm{v} \sin \mathrm{i}=80 \mathrm{kms}^{-1}$, in good agreement with photometrically derived estimates.

The abundance analysis indicates a 2 dex overabundance of Si. No significant enhancement of the iron-peak elements was found. The light elements, like $\mathrm{He}, \mathrm{C}, \mathrm{Mg}$, are underabundant by a factor of about 10 , and even more so in the case of $\mathrm{He}$. The heavy elements, like $\mathrm{Sr}, \mathrm{Y}$, and $\mathrm{Zr}$ are overabundant and follow the even-odd abundance pattern.

A detailed spectroscopic analysis of ET is being prepared for publication (Kuschnig et al. 1994, A\&A in prep.)

\section{Silicon and Helium surface mapping}

A series of 170 AURELIE spectra was available for mapping ET And. Instead of using the approximation formula for local line profiles we synthesized the spectral region for $\mathrm{Si}(4194 \AA$ to $4206 \AA)$, and for He $(4022 \AA$ to $4027 \AA)$. The inversion from observed line profiles to the surface distribution was done with an interpolation in order to derive the local line profiles for any given point on the stellar surface. An angle of inclination $i=80^{\circ}$ gave the best fit to the observed line profiles.

The resulting image for silicon shows a strong equatorial spot-like concentration. The structure in longitude is particularly well reproduced because of the coverage of 20 phases. The largest local overabundance found is about 4 dex compared to solar.

The helium map is essentially defined by two spots close to the equator. They are found in a region were silicon is relatively depleted. Only in the two strongest He spots comes the abundance close to solar.

Also this part of our investigation is being prepared for publication (Piskounov et al. 1994, A\&A in prep.).

\section{Acknowledgments}

This project was supported in Austria by the FWF (projects No. 6927 and 8776), the BMfWF (project Modellieren von radialen und nicht-radialen Pulsationen), the OAAW (project Asteroseismology), in USA by NSF grant No. AST-8716971 and the U.Hawaii (Mauna Kea Observatory), in China by NNSF grant No. F-108. The authors greatfully acknowledge telescope time allocated to this project from their institutions. 

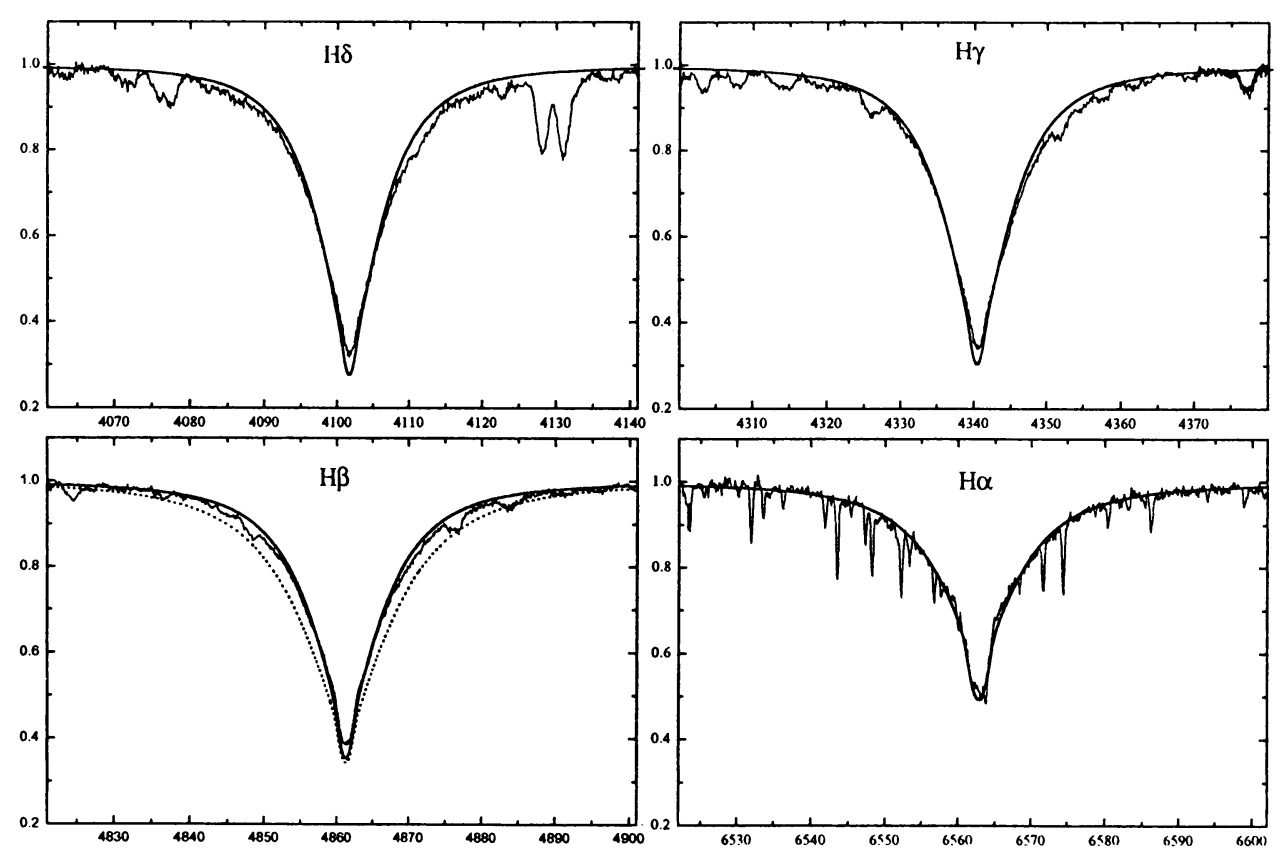

Fig. 1. Comparison of observed and computed Balmer line profiles $\left(T_{\text {eff }}=11500 \mathrm{~K}\right.$, $\log \mathrm{g}=3.5$ (solid line), $\log \mathrm{g}=4.0$ (dotted)

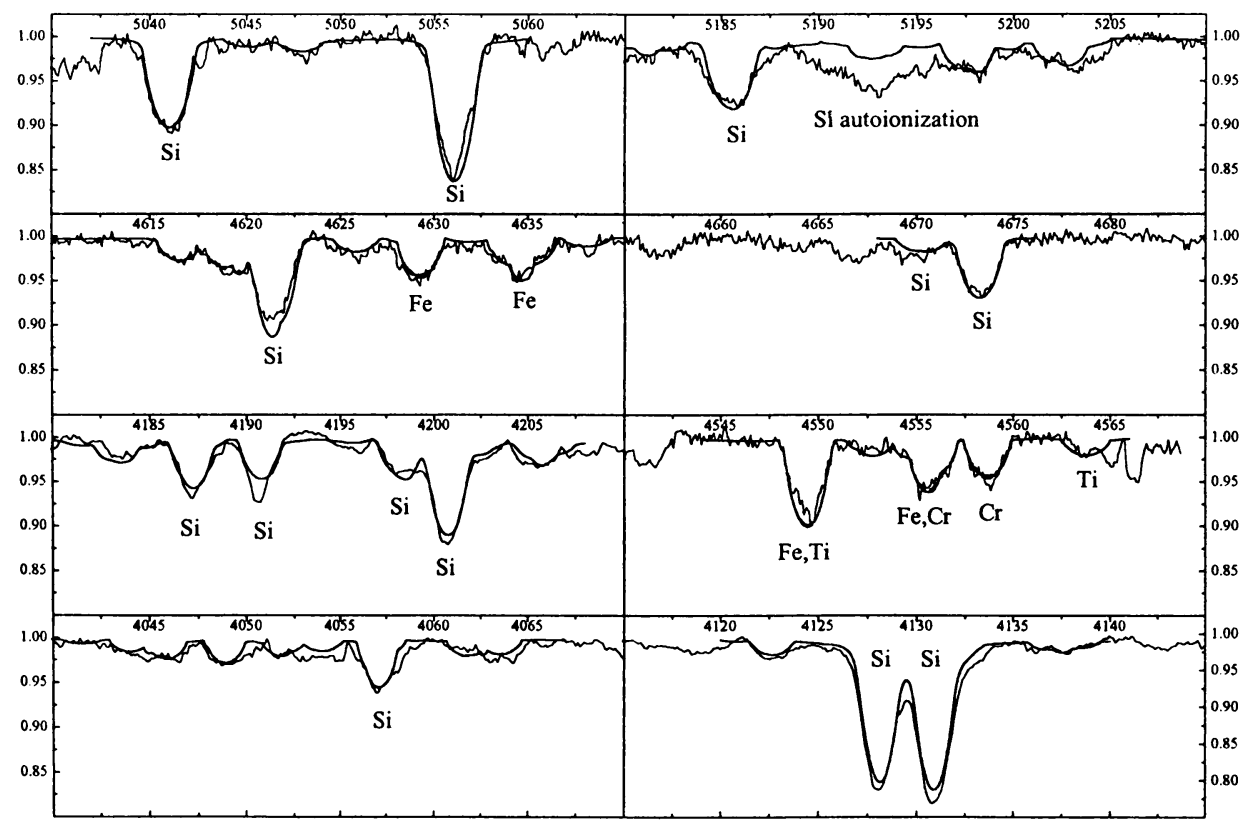

Fig. 2. Synthesized spectral regions, $T_{\text {eff }}=11500, \log \mathrm{g}=3.5, \mathrm{v} \operatorname{sini}=80 \mathrm{kms}^{-1}$ 

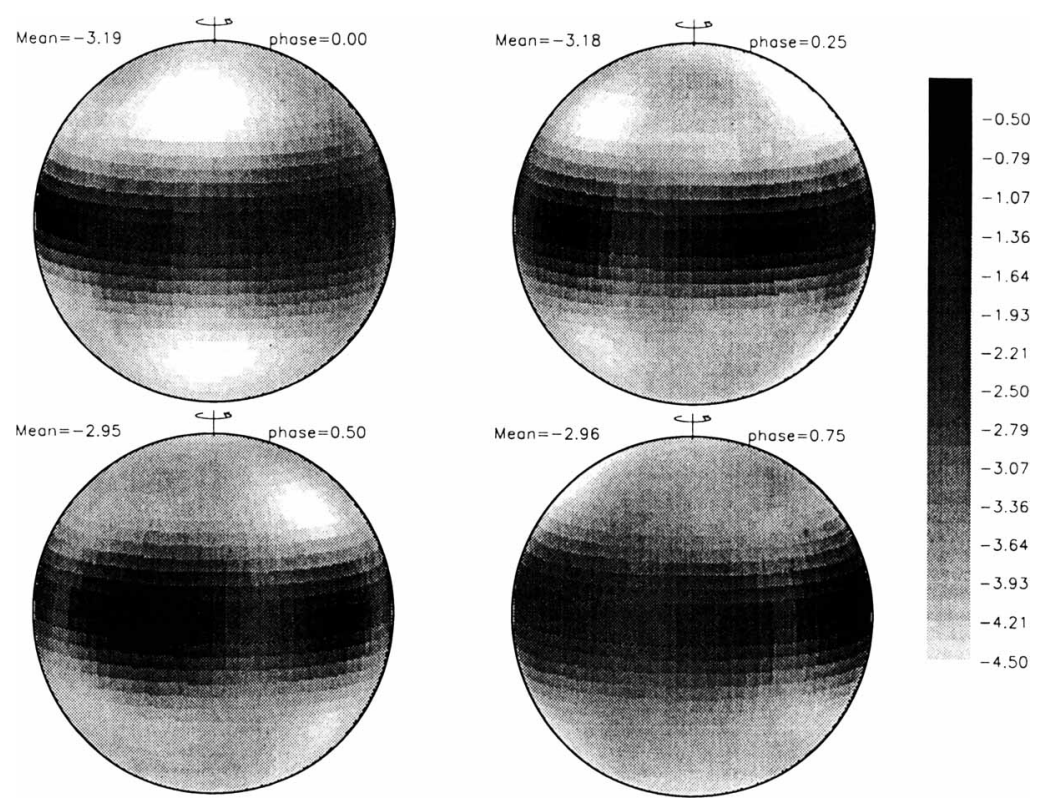

Fig. 3. Silicon surface distribution map for ET And
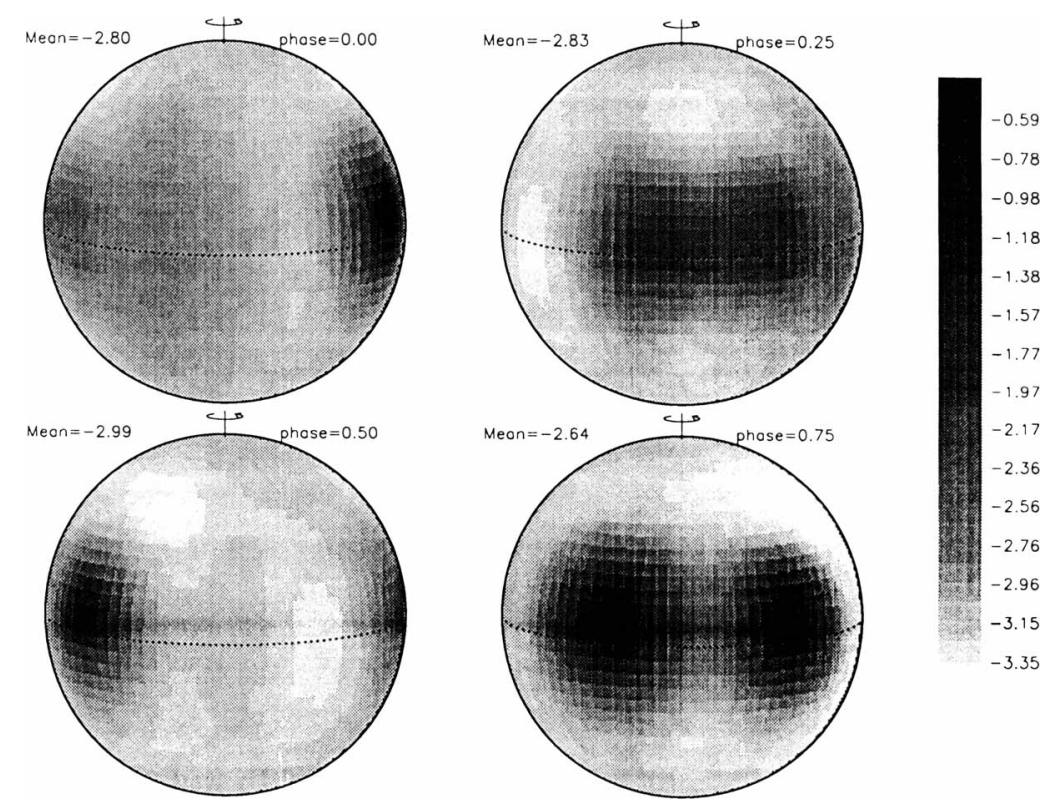

Fig. 4. Helium surface distribution map for ET And 